\title{
Imagens sociais de famílias com filhos em acolhimento e em contexto familiar: um estudo entre Brasil e Portugal
}

\section{Social images of families with children in sheltered care and in family context: a study between Brazil and Portugal}

Imágenes sociales de familias con hijos en acogimiento residencial y en contexto familiar: un estudio entre Brasil y Portugal

\section{Jana Gonçalves Zappe*}

Universidade Federal de Santa Maria, Santa Maria, Rio Grande do Sul, Brasil

\section{Naiana Dapieve Patias**}

Faculdade Meridional - IMED, Passo Fundo, Rio Grande do Sul, Brasil

\section{Joana Nunes Patrício***}

Instituto Universitário de Lisboa - ISCTE-IUL, Lisboa, Portugal

\section{Maria Manuela Calheiros****}

Instituto Universitário de Lisboa - ISCTE-IUL, Lisboa, Portugal

\section{Margarida Vaz Garrido*****}

Instituto Universitário de Lisboa - ISCTE-IUL, Lisboa, Portugal

\section{Diniz Lopes******}

Instituto Universitário de Lisboa - ISCTE-IUL, Lisboa, Portugal

\section{Débora Dalbosco Dell Aglio*******}

Universidade Federal do Rio Grande do Sul - UFRGS, Porto Alegre, Rio Grande do Sul, Brasil

\begin{abstract}
RESUMO
A literatura indica que as famílias de crianças e jovens em acolhimento institucional podem ser estigmatizadas socialmente, gerando um impacto negativo no seu bem-estar, na construção da sua identidade e no sucesso da intervenção familiar. No entanto, poucos estudos investigam empiricamente esta imagem social. Este estudo teve como objetivo analisar a imagem social das famílias de crianças e jovens em diferentes contextos em Portugal e no Brasil. Uma amostra de 378 participantes (176 portugueses e 202 brasileiros) foi solicitada a indicar cinco atributos de famílias de crianças e jovens em acolhimento institucional e outros cinco atributos de famílias de
\end{abstract}


Jana Gonçalves Zappe, Naiana Dapieve Patias, Joana Nunes Patrício, Maria Manuela Calheiros, Margarida Vaz Garrido, Diniz Lopes,

Débora Dalbosco Dell Aglio

crianças e jovens em contexto familiar, de estatutos socioeconômicos baixo e médio. Os dados indicam que em ambos os países existe um predomínio de atributos negativos associados às famílias de crianças e jovens em acolhimento institucional e de estatuto socioeconômico baixo, e um predomínio de atributos positivos associados às famílias em contexto familiar de estatuto socioeconômico médio. Destaca-se a necessidade de intervenções, especialmente com profissionais que atuam junto a essa população, para conscientização sobre essas imagens sociais.

Palavras-chave: imagens, acolhimento, estigmatização, famílias, estatuto.

\section{ABSTRACT}

The literature indicates that families of children and adolescents in sheltered care may be socially stigmatized and this has a negative impact on their well-being, the construction of their identity, and the success of family interventions. However, only a few studies have empirically investigated this social image. This study aimed to analyse the social image of the families of children and adolescents in different contexts in Portugal and Brazil. A sample of 378 participants (176 Portuguese and 202 Brazilian) was asked to indicate five attributes of families of children and adolescents in shelter care, and other five attributes of families of children and adolescents in the family context, varying from low to middle socioeconomic status. The data indicate that in both countries there is a predominance of negative attributes associated with families of children and adolescents in shelter care and low socioeconomic status, whereas a predominance of positive attributes was associated with families of children and adolescents in family context and middle socioeconomic status. It is highlighted the need of interventions, especially with professionals who work with this population, to bring out awareness of these social images.

Keywords: images, care, stigmatization, families, status.

\section{RESUMEN}

La literatura indica que las familias de los niños y jóvenes en acogimiento residencial son estigmatizadas socialmente y esto tiene un impacto negativo en su bienestar, en la construcción de su identidad y en el éxito de la intervención familiar. Sin embargo, pocos estudios han investigado empíricamente esta imagen social. Este estudio tuvo como objetivo analizar la imagen social de las familias de los niños y jóvenes en diferentes contextos en Portugal y Brasil. Se le pidió a una muestra de 378 participantes (176 portugueses y 202 brasileños) para indicar cinco atributos de las familias de niños y jóvenes en acogimiento residencial y otros cinco atributos de las familias de niños y jóvenes en el contexto familiar, nivel socioeconómico bajo y medio. Los datos indican que en ambos países hay un predominio de los atributos negativos asociados a las familias de niños y jóvenes en acogimiento residencial y el estatus socioeconómico bajo, y un predominio de los atributos positivos asociados al nivel socioeconómico promedio de las familias. Se destaca la necesidad de la intervención, sobre todo con los profesionales que trabajan con esta población, a la concientización con respecto a estas imágenes sociales.

Palabras-clave: imágenes, acogimiento, estigmatización, familias, status. 


\section{Introdução}

A família tem sido valorizada como o principal contexto de desenvolvimento durante a infância e a adolescência, tendo um papel importante na socialização primária e na constituição dos indivíduos. Em decorrência disso, diversos documentos legais nacionais e internacionais buscam incentivar, reforçar e garantir a convivência familiar, sendo que o afastamento da família é admitido apenas a título de excepcionalidade e brevidade (Brasil, 1990; 2006; 2009; Organização das Nações Unidas, 1989; Portugal, 1999; 2007).

Tanto no Brasil, como em Portugal, existem leis específicas para garantir a proteção das crianças e adolescentes e a convivência familiar. No Brasil, os princípios da Convenção Internacional dos Direitos da Criança foram adotados através do Estatuto da Criança e do Adolescente (ECA) em 1990, o qual possui como principal diretriz a Doutrina da Proteção Integral e estabelece uma série de medidas que visam garantir o desenvolvimento saudável e a convivência familiar e comunitária (Brasil, 1990). A partir do ECA, a legislação e as políticas públicas têm sido progressivamente revistas e modificadas no Brasil, apresentando evoluções significativas no sentido de romper com a cultura de institucionalização que vigorava no país ao considerar o afastamento familiar como a principal medida a ser tomada, inserindo crianças e adolescentes em instituições inadequadas ao seu desenvolvimento por longos períodos de tempo (Rizzini, \& Rizzini, 2004).

Além do ECA, outros documentos legais também contribuíram para superar a cultura de institucionalização, destacando-se neste sentido as diretrizes previstas no Plano Nacional de Convivência Familiar e Comunitária (Brasil, 2006) e na Lei da Adoção (Brasil, 2009). O Plano Nacional de Convivência Familiar e Comunitária constituiu-se como um marco nas políticas públicas brasileiras ao buscar o fortalecimento do paradigma da proteção integral e da preservação dos vínculos familiares e comunitários, colaborando para a superação do padrão tradicional de institucionalização ao instituir um novo modelo de acolhimento, com unidades menores e atendimento personalizado. A Lei da Adoção, por sua vez, inovou ao modificar o próprio ECA (Brasil, 1990), com o estabelecimento de tempo máximo para a institucionalização, especificação de atribuições das equipes técnicas dos acolhimentos, e destaque ao papel da família extensa e da importância do processo de reinserção familiar, entre outras inovações. Além disso, reiterou 0 entendimento de que a institucionalização não deve ser uma medida inicial diante da ameaça ou violação dos direitos da criança e do adolescente, incentivando a adoção de outras medidas sempre que possível (Siqueira, 2012).

Em Portugal, a Lei de Proteção a Crianças e Jovens em Perigo (Portugal, 1999), fundamentada pela Convenção dos Direitos da 
Criança, refere os princípios relativos ao superior interesse da criança e à obrigatoriedade do Estado em prestar proteção àquelas crianças privadas do seu ambiente familiar de origem. Essa proteção dá-se através de cuidados alternativos adequados que podem passar por apoio junto dos pais, junto de outro familiar, apoio para a autonomia de vida, acolhimento familiar e ainda por acolhimento em instituição. Desta forma, assim como no Brasil, o acolhimento pode ser adotado com o intuito de proteger crianças e adolescentes que se encontram em risco pessoal ou social.

Adicionalmente, também em Portugal o sistema de acolhimento institucional tem sofrido melhorias contínuas no sentido de prestar uma resposta de maior qualidade e ser capaz de promover um desenvolvimento saudável nas crianças e jovens acolhidos. De fato, ao longo do tempo, e sobretudo na década de 1980, as grandes instituições foram substituídas por instituições menores, de tipo mais familiar (Calheiros, Seabra, \& Fornelos, 1993). Já na década de 1990 foram adotadas normas, convenções e legislações que permitiram estruturar o sistema de proteção a crianças e jovens, dotando as instituições de princípios orientadores da sua ação bem como de equipes técnico-educativas que permitem uma resposta mais apropriada às necessidades das crianças e jovens em acolhimento (Convenção dos Direitos da Criança, 1990; Portugal, 1999).

Mais recentemente, em Portugal, houve a implementação do plano DOM (Desafios, Oportunidades e Mudanças; Portugal, 2007) com o objetivo de melhorar as instituições de acolhimento através da qualificação dos recursos humanos (com formação e supervisão) e da desinstitucionalização das crianças e jovens. Em 2012, esse plano evoluiu para o Plano SERE + (Sensibilizar, Envolver, Renovar, Esperança, MAIS) com o intuito de obter uma intervenção integrada e mais especializada, na garantia e melhoria da promoção de direitos e proteção das crianças e jovens acolhidos, no menor tempo de institucionalização possível (Portugal, 2012).

No entanto, embora as políticas públicas visem a melhoria das instituições e o menor tempo de institucionalização, tanto no Brasil, como em Portugal, ainda se encontram muitas crianças e jovens em acolhimento e o tempo de institucionalização ainda é prolongado. No que se refere à realidade brasileira, um levantamento concluído em 2011 identificou 36.929 crianças e adolescentes acolhidos, sendo os principais motivos de ingresso nos serviços de acolhimento a negligência, o abandono e a dependência química ou alcoólica dos pais ou responsáveis. No entanto, a existência de vínculo familiar foi constatada para a maior parte das crianças e adolescentes acolhidos $(61 \%)$, sendo que um total de $59,4 \%$ das crianças e adolescentes recebia visitas de familiares durante o período de acolhimento (Constantino, Assis, \& Mesquita, 2013). Neste sentido, é possível 
questionar se o acolhimento seria mesmo necessário com relação aos casos em que foi constatada a existência de vínculos familiares.

Outro aspecto que leva a questionamentos neste sentido é o tempo de institucionalização das crianças e adolescentes. No Brasil, as crianças e adolescentes permanecem em média 24,2 meses em acolhimento institucional, sendo que o tempo de acolhimento é mais elevado em instituições mais antigas: as mais recentes, com até cinco anos de existência, apresentam tempo médio de acolhimento de quinze meses; as que existem entre onze e vinte anos têm média de permanência de 25 meses; e entre aquelas com mais de trinta anos de vida o tempo de acolhida é, em média, de 33 meses (Constantino et al., 2013).

Em Portugal, embora os dados mais recentes indiquem uma redução do número de crianças e jovens acolhidos nos últimos anos, ainda é grande o número de crianças e adolescentes que estão sob tutela do estado. Por exemplo, o relatório de caracterização anual da situação de acolhimento das crianças e jovens de 2013 (Instituto da Segurança Social, ISS, 2014) indica que há no país 8.445 acolhidos, sendo $56,2 \%$ adolescentes (de 12 a 17 anos), de ambos os sexos, com ligeira prevalência de meninos (51,3\%) (ISS, 2014). Relativamente à situação de perigo que levou à situação atual de acolhimento da criança e jovem, destaca-se largamente a falta de supervisão e acompanhamento familiar, a exposição a modelos parentais desviantes e a negligência dos cuidados de educação e saúde (ISS, 2014). Relativamente ao tempo de acolhimento, verificase que das crianças e jovens que cessaram o acolhimento nesse mesmo ano, 40,6\% estavam em acolhimento há um ano ou menos, no entanto $37,2 \%$ estavam em acolhimento há quatro ou mais anos (ISS, 2014). Quanto ao contato com as famílias, este ganha especial relevância se considerarmos que existe uma elevada percentagem de crianças e jovens cujo projeto de vida é efetivamente a reintegração familiar (31,95\%, ISS, 2014).

Esses aspectos parecem indicar a permanência, no imaginário social, da cultura de institucionalização. No entanto, outras características e problemas estruturais do Brasil e de Portugal estão relacionadas ao afastamento de crianças e adolescentes do ambiente familiar, tais como precariedade de renda, baixas condições de trabalho e falta de oportunidades sociais e econômicas para o desenvolvimento humano. Isso significa que, nos dois países, a institucionalização de crianças e adolescentes ocorre com certa frequência em função da condição socioeconômica, na medida em que a pobreza é um dos principais aspectos relacionados com a negligência parental e consequente aplicação dessa medida (Calheiros, 2006; Cavalcante, Costa Silva, \& Magalhães, 2010; Marzol, Bonafé, \& Yunes, 2012; Siqueira \& Dell'Aglio, 2006; Vasconcelos, Yunes, \& Garcia, 2009). 
De fato, em alguns casos a pobreza pode se constituir num fator de risco ao desenvolvimento humano, na medida em que leva pais e/ou responsáveis a dispensarem menos tempo e recursos às atividades cotidianas com seus filhos, gerando falhas no exercício da parentalidade ou omissão no cumprimento de suas funções (Calheiros, 2006; Cavalcante et al., 2010). No entanto, mesmo nesses casos, a institucionalização deve ser repensada para dar lugar a medidas alternativas, como programas de transferência ou geração de renda familiar, e inserção das famílias em programas de apoio e orientação parental com vista à preservação familiar (Calheiros, Graça, \& Patrício, 2014; Rodrigo, Maiquez, Martin, \& Byrne, 2008).

O elevado número de crianças e adolescentes acolhidos institucionalmente, bem como o tempo prolongado de institucionalização podem estar relacionados, também, com o modo como as famílias e as comunidades de origem desses jovens são percebidas. No levantamento concluído em 2011 no Brasil (Constantino et al., 2013), foi observado que a visão que os profissionais que atuam nos serviços de acolhimento institucional possuem sobre as famílias das crianças e adolescentes acolhidos é muitas vezes negativa. Os profissionais destacaram a presença frágil ou danosa das famílias, a existência de conflitos e especialmente desinteresse pelos filhos. É interessante notar que os mesmos profissionais apontam em seu discurso a inexistência de vínculos e que as visitas aos filhos são raras. No entanto, os dados quantitativos apontaram para a existência de vínculos e a realização de visitas para a maioria dos casos, o que indica a presença de imagens negativas possivelmente mais vinculadas à cultura de institucionalização ainda presente no imaginário social do que à realidade propriamente dita. É digno de nota que estas imagens negativas levam ao descrédito em relação às famílias dos acolhidos e dificultam o trabalho no sentido do fortalecimento dos vínculos que darão suporte à reintegração familiar (Constantino et al., 2013).

Outros estudos também apontam que a presença de imagens sociais negativas associadas às famílias de origem das crianças e jovens institucionalizados prejudica o trabalho no sentido da reinserção familiar e comunitária. Um estudo de caso que investigou os processos de interação entre uma instituição de acolhimento e a família de uma criança institucionalizada revelou que a relação entre a família e a instituição tem por base sentimentos de desconfiança mútua. Tal padrão de interação dificultou e prorrogou a tentativa de reinserção da criança no ambiente familiar e interferiu diretamente no aumento do tempo de institucionalização (Vasconcelos et al., 2009). No mesmo sentido, outros estudos também demonstraram que a presença de imagens negativas associadas às famílias dificulta 0 trabalho e traz um obstáculo a mais à preservação dos vínculos familiares e reinserção familiar (Siqueira, Massignan, \& Dell'Aglio, 
2011; Siqueira, Tubino, Schwarz, \& Dell'Aglio, 2009; Vasconcelos et al., 2009).

Características atribuídas a diferentes grupos sociais identificam os indivíduos enquanto membros desse grupo e traduzem-se em imagens sociais. Estas, por sua vez, são ideias partilhadas sobre determinados grupos ou sociedades e prevalecem mesmo na ausência de evidência objetiva de que são reais (Corsini, 1999). Quando esses atributos ou ideias partilhadas são negativas, considera-se que há um estigma que representa a condição de uma pessoa que se encontra incapacitada para receber a aceitação social (Goffman, 1988). Dada a relevância desses processos de estigma social, e da sua repercussão na vida das crianças e adolescentes institucionalizados, torna-se importante compreender e identificar que imagens e estereótipos são associados às suas famílias.

Diante disso, considera-se fundamental estudar as imagens sociais que pessoas com e sem contato com esta população atribuem às famílias das crianças e jovens em acolhimento institucional, pois poucos estudos investigam empiricamente esta imagem social, nomeadamente de forma comparativa à imagem social de famílias em contexto normativo de nível socioeconômico médio ou baixo. A necessidade de investigar estas imagens sociais de forma sistemática levou ao estabelecimento de um convênio de cooperação entre uma equipe de investigação Brasileira e outra Portuguesa, que em conjunto desenvolveram um projeto de pesquisa no qual um dos objetivos principais era analisar o conteúdo das imagens que leigos e profissionais têm sobre as famílias de crianças e jovens em acolhimento institucional, verificando se estas incluem traços/atributos mais negativos quando comparadas com as imagens de famílias de crianças e jovens que vivem em contexto familiar.

A investigação sobre este tema pode subsidiar pesquisas e intervenções em variadas áreas, especialmente na educação inclusiva de crianças e jovens institucionalizados e no fortalecimento das redes de proteção, incluindo as famílias e comunidades. Dessa forma, este artigo teve como objetivo analisar as imagens sociais de famílias de crianças e jovens em acolhimento institucional, de famílias de nível socioeconômico baixo e de famílias de nível socioeconômico médio, comparando os resultados de uma amostra portuguesa e uma amostra brasileira.

\section{Método}

\section{Participantes}

A amostra foi constituída por 378 participantes, sendo 202 do Brasil e 176 de Portugal, com idades entre os 16 e os 77 anos $(M=33.72, D P$ 
$=12.93)$. A maior parte dos participantes é do sexo feminino $(80.1 \%)$. As duas amostras são equivalentes quanto à idade, sexo, estado civil e ao fato de ter filhos. No entanto, foram observadas diferenças no nível educacional, no rendimento mensal e no contato e trabalho com crianças e jovens em acolhimento. Concretamente (ver Tabela 1), existem mais participantes com o primeiro ciclo e com o ensino superior na amostra portuguesa do que na brasileira, e mais com ensino secundário na amostra brasileira do que na portuguesa. $\mathrm{Na}$ amostra brasileira há mais participantes com um rendimento superior a 3.000 reais e na portuguesa há mais participantes com um rendimento inferior a 2.000 euros, embora sejam equivalentes ao serem convertidos. Há também mais participantes na amostra do Brasil que conhecem ou trabalham com crianças e jovens em risco do que na amostra portuguesa.

Tabela 1

Dados Sociodemográficos da Amostra

\begin{tabular}{|c|c|c|c|c|}
\hline Variável & $\begin{array}{c}\text { Portugal } \\
\mathrm{n}=176\end{array}$ & $\begin{array}{c}\text { Brasil } \\
n=202\end{array}$ & $\begin{array}{l}\text { Teste } t \text { / Qui- } \\
\text { quadrado }\end{array}$ & $p$ \\
\hline Idade: Média (DP) & $\begin{array}{c}34.66 \\
(14.03)\end{array}$ & $\begin{array}{c}32.76 \\
(11.67)\end{array}$ & $t(340)=-1.359$ & .175 \\
\hline Sexo Feminino (\%) & 80.57 & 79.60 & $x^{2}(1)=0.055$ & .814 \\
\hline Estado civil (\%) & & & $x^{2}(4)=4.499$ & .343 \\
\hline Solteiro & 59.54 & 54.21 & & \\
\hline Casado/União de facto & 35.26 & 37.37 & & \\
\hline Divorciado & 4.05 & 7.37 & & \\
\hline Viúvo & 1.16 & 1.05 & & \\
\hline Tem filhos $(\%)$ & 35.47 & 44.62 & $x^{2}(1)=3.180$ & .075 \\
\hline Habilitações (\%) & & & $x^{2}(4)=18.232$ & .001 \\
\hline $1^{\circ}$ ciclo & 4.12 & 0.00 & & \\
\hline $2^{\circ}$ ciclo & 1.18 & 0.00 & & \\
\hline $3^{\circ}$ ciclo & 4.71 & 4.06 & & \\
\hline Ensino Secundário & 32.94 & 49.24 & & \\
\hline Ensino Superior & 57.06 & 46.70 & & \\
\hline $\begin{array}{l}\text { Rendimento mensal do } \\
\text { agregado }(\%)\end{array}$ & & & $x^{2}(4)=135.327$ & $<.001$ \\
\hline$<1000 € /$ RS & 21.37 & 1.23 & & \\
\hline $1000-2000 € / R \$$ & 43.59 & 5.56 & & \\
\hline $2000-3000 € / R \$$ & 24.79 & 19.75 & & \\
\hline $3000-4000 \mathrm{C} / \mathrm{R} \$$ & 7.69 & 25.31 & & \\
\hline$>4000 € / R \$$ & 2.56 & 48.15 & & \\
\hline $\begin{array}{l}\text { Conhece crianças/jovens } \\
\text { em acolhimento }(\%)\end{array}$ & 34.29 & 56.92 & $x^{2}(1)=19.014$ & $<.001$ \\
\hline $\begin{array}{l}\text { Trabalha na área de } \\
\text { crianças/jovens em perigo } \\
(\%)\end{array}$ & 13.71 & 42.93 & $x^{2}(1)=38.335$ & $<.001$ \\
\hline
\end{tabular}

\section{Instrumentos}

Foi aplicado um questionário de resposta aberta, no qual era solicitado aos participantes que indicassem cinco atributos/características de famílias de crianças (entre 0 e 12 anos) 
ou de jovens (entre 12 e 18 anos) em acolhimento institucional e outros cinco atributos para famílias de crianças (entre 0 e 12 anos) ou de jovens (entre 12 e 18 anos) em contexto familiar (alternando o estatuto socioeconômico - SES, da família entre baixo e médio).

Nos questionários, as famílias foram descritas da seguinte maneira (versão em português do Brasil):

Família de SES baixo - Imagine uma família de três pessoas, em que, um ou os dois pais estão desempregados e não possuem veículo próprio. Em termos de escolaridade, estes pais têm, no máximo, o ensino fundamental. Esta família tem uma moradia pouco confortável e com condições deficitárias.

Família de SES médio - Imagine uma família de três pessoas, em que os dois pais têm emprego e veículo próprio. Em termos de escolaridade, estes pais têm, no mínimo, o ensino médio completo. Esta família tem uma moradia confortável e com boas condições.

Família com filhos em acolhimento - O acolhimento em Abrigos constitui uma das medidas de proteção e de garantia dos direitos fundamentais das crianças e dos jovens que, no seu meio natural de vida, estão expostos a condições adversas para 0 seu desenvolvimento. Esta medida de acolhimento em instituição consiste na colocação da criança ou jovem aos cuidados de uma entidade que disponha de instalações e equipamento de acolhimento permanente e de uma equipe técnica, oferecendo cuidados adequados e condições que permitam a sua educação, bem-estar e desenvolvimento integral. Foram desenvolvidas diferentes versões do questionário que se referiam a quatro condições: 1) Família com criança em acolhimento Vs Família com criança de SES médio $(n=90) ; 2)$ Família com criança em acolhimento Vs Família com criança de SES baixo $(n=85) ; 3)$ Família com jovem em acolhimento Vs Família com jovem de SES médio $(n=109)$; 4) Família com jovem em acolhimento Vs Família com jovem de SES baixo $(n=94)$. As quatro versões do questionário foram distribuídas aleatoriamente aos participantes.

\section{Procedimentos}

O projeto de investigação foi aprovado por Comitês de Ética nos dois países. Os participantes foram recrutados em diferentes instituições de intervenção na infância (e.g., acolhimento institucional, Comissões de Proteção de crianças e jovens em risco), em instituições de ensino superior e formação pós-graduada e outros locais de circulação pública. A amostra deste estudo foi composta por conveniência, sobretudo nos grandes centros urbanos de Porto Alegre e Lisboa, não sendo representativa da população brasileira e portuguesa. Foram oferecidas informações sobre os objetivos do estudo, sobre a voluntariedade da participação, a garantia de sigilo das informações pessoais e a possibilidade de desistência do estudo a qualquer 
momento. Os participantes que concordaram em participar assinaram o Termo de Consentimento Livre e Esclarecido.

\section{Análise de Dados}

O procedimento utilizado foi semelhante ao utilizado em outros estudos (e.g., Carneiro, Albuquerque, \& Fernandez, 2008; Kuznetsova, 2005; Massey, 2010; Nario-Redmond, 2010; Calheiros, Garrido, Lopes, \& Patrício, 2015). Inicialmente foi elaborada uma lista dos atributos legíveis referidos pelos participantes, sendo que esta foi composta por 779 atributos na amostra portuguesa e por 1085 atributos na brasileira. Em seguida, os atributos foram agregados em equipe tendo em consideração a uniformidade na escrita (e.g., revoltado com revoltados) e os sinônimos dos atributos (e.g., triste com infeliz), o que reduziu a lista inicial para 85 atributos na amostra portuguesa e 84 atributos na brasileira. Por fim, foram excluídos das análises posteriores os atributos referidos três vezes ou menos. Assim a lista final de atributos para análise ficou reduzida a 74 atributos na amostra portuguesa e a 73 atributos na brasileira.

No que respeita à análise destes atributos, foram selecionados os atributos referidos por pelo menos $10 \%$ da amostra de cada um dos países. Em seguida, efetuamos uma análise descritiva tendo como referência os 10 atributos mais referidos para cada tipo de família em ambos os países. Para comparar as imagens sociais destas famílias em Portugal e no Brasil testamos as diferenças na proporção com que estes atributos foram referidos através de análises discriminantes. Por fim, verificamos se a imagem social destas famílias difere em função do contato dos participantes com esta população, também através de análises discriminantes.

\section{Resultados}

\section{As Imagens Sociais de Famílias de Crianças e Jovens em Acolhimento}

A análise dos dez atributos mais utilizados para descrever as famílias de crianças e jovens em acolhimento indica que os atributos mais referidos pela amostra brasileira foram baixa escolaridade, consumos/abusos, desamparada/carenciada, desapegada emocionalmente, desestruturada, desorganizada, desprotetora, irresponsável, negligente e violenta (Tabela 2). Na amostra portuguesa os atributos mais referidos foram desamparada/carenciada, desestruturada, desinteressada, desorganizada, instável, irresponsável, negligente, problemática, triste e violenta. Assim, em ambos os países foram salientados 
apenas atributos negativos, relativos à falta de estrutura, organização, recursos e apoio destas famílias, bem como a sua irresponsabilidade e práticas parentais disfuncionais (i.e., desamparada/carenciada, desestruturada, desorganizada, irresponsável, negligente e violenta).

A análise discriminante para a descrição das famílias de crianças e jovens em acolhimento é significativa (Eigenvalue $=.656$, Canonical $\mathrm{R}=.629$, Wilks' Lambda $\left.=.604, \mathrm{X}^{2}(19)=184.927, p<.001\right)$. Esta função discriminante classifica corretamente $77 \%$ da amostra (Classification statistics Brasil $=76.7 \%$, Portugal $=77.3 \%$; Canonical discriminant functions at group centroids Brasil $=-.754$, Portugal $=$ .866) e indica que os participantes portugueses usam mais os atributos triste, problemática, disfuncional, instável e desinteressada para descrever estas famílias, enquanto os participantes brasileiros usam mais os atributos violenta, consumos/abusos, sem valores sociais, desprotetora, desestruturada, desapegada emocionalmente, vulnerável (Tabela 2).

Tabela 2

Análise de Frequências e Discriminante para as Familias de Crianças/jovens em Acolhimento

\begin{tabular}{lccc}
\hline & $\begin{array}{c}\text { Brasil } \\
(\%)\end{array}$ & $\begin{array}{c}\text { Portugal } \\
(\%)\end{array}$ & $\begin{array}{c}\text { Standardized canonical } \\
\text { discriminant function } \\
\text { coefficients }\end{array}$ \\
\hline Triste & 6.9 & $\mathbf{2 1 . 6}$ & $.359^{* * * *}$ \\
Problemática & 3.5 & $\mathbf{1 8 . 2}$ & $.324^{* * * *}$ \\
Disfuncional & 0.0 & 12.5 & $.312^{* * * *}$ \\
Instável & 3.5 & $\mathbf{1 8 . 2}$ & $.306^{* * * *}$ \\
Desinteressada & 4.0 & $\mathbf{1 4 . 2}$ & $.189^{* * * *}$ \\
Sem recursos & 8.9 & 10.8 & .117 \\
Irresponsável & $\mathbf{1 1 . 9}$ & $\mathbf{1 8 . 2}$ & .116 \\
Negligente & $\mathbf{1 4 . 9}$ & $\mathbf{1 7 . 0}$ & .093 \\
Desamparada & $\mathbf{3 8 . 6}$ & $\mathbf{4 2 . 0}$ & .088 \\
Desorganizada & $\mathbf{1 2 . 9}$ & $\mathbf{1 4 . 2}$ & .059 \\
Carente & 11.4 & 11.9 & .037 \\
Baixa escolaridade & $\mathbf{1 4 . 9}$ & 10.8 & .034 \\
Violenta & $\mathbf{2 5 . 7}$ & $\mathbf{1 5 . 9}$ & $-.089^{* *}$ \\
Consumos/abusos & $\mathbf{2 1 . 3}$ & 11.4 & $-.130^{*}$ \\
Sem valores sociais & 10.4 & 0.0 & $-.257^{* * * *}$ \\
Desprotetora & $\mathbf{1 2 . 9}$ & 0.0 & $-.304^{* * * *}$ \\
Desestruturada & $\mathbf{3 6 . 1}$ & $\mathbf{1 9 . 9}$ & $-.339^{* * * *}$ \\
Desapegada & $\mathbf{1 4 . 9}$ & 0.0 & $-.360^{* * * *}$ \\
emocionalmente & 10.9 & 0.0 & $-.433^{* * * *}$ \\
Vulnerável & &
\end{tabular}

Nota: Em negrito os 10 atributos mais referidos.

$* p<.05,{ }^{* *} p<.01,{ }^{* * *} p<.001$ 


\section{As Imagens Sociais de Famílias de SES Baixo}

No que diz respeito às famílias de SES baixo, os atributos mais referidos pela amostra brasileira foram acomodada/conformada, angustiada, baixa escolaridade, batalhadora/esforçada, com dificuldades, conflituosa, desamparada/carenciada, desestruturada, preocupada, sem perspectiva, triste e unida (Tabela 3). Os atributos mais referidos pela amostra portuguesa foram angustiada, batalhadora/esforçada, desamparada/carenciada, desesperada, desmotivada, preocupada, triste, unida, humilde, insegura, revoltada. Analisando os atributos comuns a ambos os países verificamos que foram salientados sobretudo atributos negativos relativos à falta de recursos e apoio destas famílias (i.e., desamparada/carenciada), bem como ao mal-estar emocional (i.e., angustiada, preocupada, triste). Contudo, salienta-se que em ambos os países foram destacados dois atributos positivos (i.e., batalhadora/esforçada e unida). Salienta-se ainda que, ao contrário do que se verificou para as famílias de crianças e jovens em acolhimento, apesar de serem utilizados atributos relativos a problemas no funcionamento e estrutura familiar (e.g., desestruturada, desprotetora) para estas famílias, não são referidas práticas parentais disfuncionais (como negligente e violenta).

A análise discriminante para a descrição das famílias de crianças e jovens de SES baixo é significativa (Eigenvalue $=.294$, Canonical $\mathrm{R}=$ .477, Wilks' Lambda $\left.=.773, \mathrm{x}^{2}(21)=94.340, \mathrm{p}<.001\right)$. Esta função discriminante classifica corretamente $67.2 \%$ da amostra (Classification statistics Brasil $=57.9 \%$, Portugal $=77.8 \%$;

Canonical discriminant functions at group centroids Brasil $=.505$, Portugal $=-.580)$ e indica que os participantes portugueses usam mais os atributos desesperada, desmotivada, preocupada, triste, unida e revoltada para descrever estas famílias, enquanto os participantes brasileiros usam mais os atributos afetuosa, com dificuldades, desestruturada, desprotetora, e sem perspectiva (Tabela 3). 
Tabela 3

Análise de Frequências e Discriminante para as Familias de SES Baixo

\begin{tabular}{|c|c|c|c|}
\hline & $\begin{array}{c}\text { Brasil } \\
(\%)\end{array}$ & $\begin{array}{l}\text { Portugal } \\
(\%)\end{array}$ & $\begin{array}{c}\text { Standardized canonical } \\
\text { discriminant function } \\
\text { coefficients }\end{array}$ \\
\hline Desprotetora & 10.2 & 0.0 & $.447^{* * * *}$ \\
\hline Desestruturada & 19.3 & 2.2 & $.436^{* * * *}$ \\
\hline Afetuosa & 10.2 & 6.6 & $.331^{* * *}$ \\
\hline Sem perspectiva & 11.4 & 0.0 & $.262^{* * *}$ \\
\hline Com dificuldades & 13.6 & 5.5 & $.112^{*}$ \\
\hline Angustiada & 15.9 & 16.5 & .174 \\
\hline Conflituosa & 12.5 & 5.5 & .132 \\
\hline $\begin{array}{l}\text { Batalhadora/esforça } \\
\text { da }\end{array}$ & 11.4 & 18.7 & .120 \\
\hline $\begin{array}{l}\text { Acomodada/conform } \\
\text { ada }\end{array}$ & 15.9 & 9.9 & .100 \\
\hline Carente & 10.2 & 7.7 & .041 \\
\hline Baixa escolaridade & 11.4 & 8.8 & .031 \\
\hline Insegura & 6.8 & 12.1 & -.041 \\
\hline Sem recursos & 10.2 & 5.5 & -.042 \\
\hline $\begin{array}{l}\text { Desamparada/caren } \\
\text { ciada }\end{array}$ & 43.2 & 35.2 & -.121 \\
\hline Humilde & 8.0 & 12.1 & -.168 \\
\hline Preocupada & 13.6 & 24.2 & $-.138^{*}$ \\
\hline Triste & 13.6 & 29.7 & $-.205^{* *}$ \\
\hline Unida & 12.5 & 16.5 & $-.209^{*}$ \\
\hline Revoltada & 0.0 & 15.4 & $-.330^{* * * * *}$ \\
\hline Desesperada & 0.0 & 12.1 & $-.234^{* * * *}$ \\
\hline Desmotivada & 3.4 & 17.6 & $-.342^{* * * *}$ \\
\hline
\end{tabular}

Nota: Em negrito os 10 atributos mais referidos. São mais de 10 quando a percentagem se repete.

$* p<.05, * * p<.01, * * * p<.001$

\section{As Imagens Sociais de Famílias de SES Médio}

No que diz respeito às famílias de SES médio, os atributos mais referidos pela amostra brasileira foram afetuosa, batalhadora/esforçada, com recursos, economicamente favorecida, educada, estruturada, feliz, organizada, protetora, responsável e trabalhadora (Tabela 4). Os atributos mais referidos pela amostra portuguesa foram afetuosa, unida, acolhedora, educada, estável, feliz, protetora, responsável, trabalhadora e segura. Assim, em ambos os países foram salientados sobretudo atributos positivos relativos às competências e bem-estar destas famílias (i.e., afetuosa, educada, feliz, protetora, responsável e trabalhadora).

A análise discriminante para a descrição das famílias de crianças e jovens de SES médio também é significativa (Eigenvalue $=.262$, Canonical $\mathrm{R}=.456$, Wilks' Lambda $=.792, \mathrm{X}^{2}(22)=85.055, \mathrm{p}<$ $.001)$. Esta função discriminante classifica corretamente $68.8 \%$ da amostra (Classification statistics Brasil $=85.1 \%$, Portugal $=50 \%$; Canonical discriminant functions at group centroids Brasil $=-.477$, 
Portugal $=.547)$ e indica que os participantes portugueses usam mais os atributos preocupada, unida, acolhedora, funcional, amigável, autoritária e segura para descrever estas famílias, enquanto os participantes brasileiros usam mais os atributos afetuosa, com recursos e economicamente favorecida (Tabela 4 ).

Tabela 4

Análise de Frequências e Discriminante para as Familias de SES Médio

\begin{tabular}{lccc}
\hline & $\begin{array}{c}\text { Brasil } \\
(\%)\end{array}$ & $\begin{array}{c}\text { Portugal } \\
(\%)\end{array}$ & $\begin{array}{c}\text { Standardized canonical } \\
\text { discriminant function } \\
\text { coefficients }\end{array}$ \\
\hline Afetuosa & $\mathbf{3 3 . 3}$ & $\mathbf{1 8 . 8}$ & $-.305^{* * *}$ \\
Batalhadora/esforçada & $\mathbf{1 5 . 8}$ & 8.2 & -.012 \\
Preocupada & 7.9 & 10.6 & $.232^{* *}$ \\
Unida & 8.8 & $\mathbf{2 0 . 0}$ & $.187^{*}$ \\
Acolhedora & 4.4 & $\mathbf{1 5 . 3}$ & $.199^{*}$ \\
Atenciosa & 8.8 & 10.6 & .133 \\
Com recursos & $\mathbf{2 0 . 2}$ & 12.9 & $-.139^{* *}$ \\
Economicamente & $\mathbf{2 2 . 8}$ & 7.1 & $-.308^{* * *}$ \\
favorecida & $\mathbf{1 9 . 3}$ & $\mathbf{2 2 . 4}$ & .087 \\
Educada & 14.0 & $\mathbf{2 9 . 4}$ & .081 \\
Estável & $\mathbf{2 1 . 9}$ & 10.6 & -.132 \\
Estruturada & $\mathbf{1 8 . 4}$ & $\mathbf{2 7 . 1}$ & .027 \\
Feliz & .9 & 10.6 & $.320^{*}$ \\
Funcional & $\mathbf{2 2 . 8}$ & 14.1 & -.153 \\
Organizada & $\mathbf{2 6 . 3}$ & $\mathbf{2 5 . 9}$ & -.118 \\
Protetora & 9.6 & 10.6 & -.065 \\
Realizada & $\mathbf{1 5 . 8}$ & $\mathbf{2 2 . 4}$ & -.016 \\
Responsável & $\mathbf{2 2 . 8}$ & $\mathbf{2 0 . 0}$ & .005 \\
Trabalhadora & 4.4 & 10.6 & .032 \\
Tranquila & 0.0 & 14.1 & $.476^{* * * *}$ \\
Amigável & 0.0 & 12.9 & $.379^{* * * *}$ \\
Autoritária & 0.0 & $\mathbf{1 5 . 3}$ & $.388^{* * * *}$ \\
Segura & & & \\
\hline Nota: Em & 0.0 &
\end{tabular}

Nota: Em negrito os 10 atributos mais referidos. São mais de 10 quando a percentagem se repete.

$* p<.05, * * *<<.01,{ }^{* * * *} p<.001$

\section{A Imagem Social de Famílias de Diferentes Contextos em Função do Contato dos Participantes com Crianças e Jovens em Acolhimento}

No que diz respeito à variação da imagem social de famílias de diferentes contextos em função do contato dos participantes com crianças e jovens em acolhimento verificamos que apenas as análises discriminantes para a descrição das famílias de crianças e jovens em acolhimento institucional foram significativas (Conhece criança/jovem em acolhimento Eigenvalue $=.162$, Canonical $\mathrm{R}=.374$, Wilks' Lambda $=.860, \mathrm{X}^{2}(19)=53.873, \mathrm{p}<.001$; Trabalha área de crianças/jovens em risco Eigenvalue $=.200$, Canonical $\mathrm{R}=.408$, 
Wilks' Lambda $\left.=.833, \mathrm{x}^{2}(19)=65.879, \mathrm{p}<.001\right)$. Na descrição das famílias de SES baixo ou médio, o contato dos participantes com crianças e jovens em acolhimento não tem qualquer efeito significativo.

Concretamente, tal como se pode observar na Tabela 5, verificamos que os participantes que conhecem crianças e jovens em acolhimento usam mais os atributos desapegada emocionalmente, desestruturada, desorganizada e sem valores sociais para descrever as suas famílias, enquanto os participantes que não conhecem crianças e jovens em acolhimento usam mais os atributos disfuncional, triste e problemática. Já os participantes que trabalham na área de crianças e jovens em risco usam mais os atributos vulnerável, negligente, desorganizada e desapegada emocionalmente para descrever as famílias de crianças e jovens em acolhimento, enquanto os participantes que não trabalham na área usam mais os atributos disfuncional, triste, problemática, instável e carente. Assim, os participantes com mais contato com esta população salientam mais atributos relativos às lacunas parentais em termos afetivos, funcionais e estruturais (e.g., desapegada emocionalmente, desorganizada, negligente, desestruturada) enquanto os outros salientam mais aspetos relativos à família em si (e.g., triste, problemática, instável, carente). 
Tabela 5

Análises discriminantes para as familias de crianças/jovens em acolhimento em função do contato com crianças/jovens em acolhimento

\begin{tabular}{|c|c|c|}
\hline Canonical discriminant functions & $\begin{array}{c}\text { Conhece } \\
\text { criança/jovem } \\
\text { em } \\
\text { acolhimento }\end{array}$ & $\begin{array}{c}\text { Trabalha área } \\
\text { de } \\
\text { crianças/joven } \\
\text { s em risco }\end{array}$ \\
\hline \multicolumn{3}{|l|}{$\begin{array}{l}\text { Standardized canonical discriminant function } \\
\text { coefficients }\end{array}$} \\
\hline Baixa escolaridade & .112 & .033 \\
\hline Carente & -.280 & $.355^{* s *}$ \\
\hline Consumos abusos & .148 & -.163 \\
\hline Desamparada/carenciada & -.198 & .130 \\
\hline Desapegada emocionalmente & $.239^{*}$ & $-.427^{* * *}$ \\
\hline Desestruturada & $.377^{*}$ & -.257 \\
\hline Desinteressada & .080 & .004 \\
\hline Desorganizada & $.433^{* * *}$ & $-.343^{*}$ \\
\hline Desprotetora & -.161 & .218 \\
\hline Instável & -.154 & $.235^{*}$ \\
\hline Irresponsável & -.049 & .200 \\
\hline Negligente & .276 & $-.386^{* * *}$ \\
\hline Problemática & $-.325^{* *}$ & $.165^{*}$ \\
\hline Sem recursos & -.220 & -.006 \\
\hline Sem valores sociais & $.199^{*}$ & -.113 \\
\hline Triste & $-.304 *$ & $.258^{*}$ \\
\hline Violenta & -.136 & .186 \\
\hline Vulnerável & .243 & $-.306^{* * *}$ \\
\hline Disfuncional & $-.259^{*}$ & $.284^{* * *}$ \\
\hline \multicolumn{3}{|l|}{$\begin{array}{l}\text { Canonical discriminant functions at group } \\
\text { centroids }\end{array}$} \\
\hline Sim & .433 & -.694 \\
\hline Não & -.372 & .287 \\
\hline \multicolumn{3}{|l|}{ Classification statistics } \\
\hline Sim & 61.4 & 65.1 \\
\hline Não & 69.8 & 70.8 \\
\hline Cases correctly classified & 65.9 & 69.2 \\
\hline
\end{tabular}

$* p<.05, * * p<.01$

\section{Discussão}

Os resultados do estudo permitiram a identificação de algumas especificidades nos atributos utilizados pela amostra brasileira e portuguesa para descrever as famílias. Na descrição das famílias de SES baixo a amostra portuguesa salientou mais aspectos de malestar e desajustamento psicológico das famílias (e.g., desesperada, desmotivada, preocupada, triste e revoltada) enquanto a amostra brasileira salientou mais aspectos estruturais e funcionais (e.g., com dificuldades, desestruturada, desprotetora). No caso das famílias de SES médio, a amostra portuguesa salientou mais aspetos estruturais e funcionais (e.g., preocupada, unida, acolhedora, funcional, amigável, autoritária e segura) enquanto a amostra brasileira 
salientou mais aspetos afetivos e econômicos (e.g., afetuosa, com recursos e economicamente favorecida). Com relação às famílias de crianças e jovens em acolhimento institucional, apesar de utilizarem palavras diferentes, ambas as amostras salientam problemas nas mesmas dimensões, i.e. funcional (disfuncional), emocional (triste, vulnerável) estrutural (instável, desestruturada), de negligência e mau trato parental (desapegada emocionalmente, desinteressada, desprotetora, violenta) e fatores de risco gerais (problemática, consumos/abusos, sem valores sociais).

Independentemente destas especificidades, este estudo permite concluir que, tanto no Brasil como em Portugal, as imagens sociais são sobretudo negativas para as famílias de crianças e jovens em acolhimento institucional e para as famílias de nível socioeconômico baixo, e positivas para as famílias de nível socioeconômico médio. De fato, as famílias de crianças e jovens em acolhimento institucional são percebidas como famílias com práticas parentais disfuncionais e sem estrutura, organização, recursos e apoio. As famílias de SES baixo são percebidas como famílias sem recursos e apoio, com alguns fatores de risco, lacunas no funcionamento familiar e mal-estar emocional, mas não são salientadas práticas parentais disfuncionais. Por fim, as famílias de SES médio são percebidas como famílias competentes e com bem-estar em diversos níveis (e.g., socioeconômico, profissional, funcional, emocional, social, parental).

As semelhanças entre as imagens sociais relacionadas com as famílias de estatuto socioeconômico baixo e com as das famílias de crianças e jovens em instituições de acolhimento podem ser explicadas pela história da institucionalização, muito vinculada a problemas estruturais tais como precariedade de renda, condições de trabalho aviltantes e falta de oportunidades sociais e econômicas para o desenvolvimento humano (Cavalcante et al., 2010; Marzol et al., 2012; Rodrigues, Barbosa-Ducharne, \& del Valle, 2013; Siqueira \& Dell'Aglio, 2006; Vasconcelos et al., 2009). Embora a pobreza possa se constituir como um fator de risco em alguns casos, ela não pode ser analisada isoladamente, mas em conjunto com outros fatores, de forma que o baixo nível socioeconômico da família não pode ser 0 único motivo para a institucionalização, sobretudo porque existem medidas alternativas que podem ser adotadas nestes casos, como programas de transferência ou geração de renda familiar, e inserção das famílias em programas de apoio e orientação parental (Calheiros, Graça \& Patrício, 2014; Rodrigo et al., 2008; Siqueira, 2012).

A identificação de imagens sociais negativas associadas a famílias de crianças e jovens em acolhimento institucional merece destaque, pois estas imagens podem se constituir como um obstáculo à implementação das políticas de preservação e valorização da convivência familiar, tanto no Brasil como em Portugal (Brasil, 1990; 2006; 2012; Portugal, 1999; 2007; 2012). Estas famílias foram 
descritas com atributos negativos, relativos à falta de estrutura, organização, recursos e apoio, bem como à sua irresponsabilidade e práticas parentais disfuncionais, o que demonstra empiricamente 0 que outros estudos já apontaram, embora não tenham focado diretamente as imagens sociais (Constantino et al., 2013; Sykes, 2011; Yunes \& Szymanski, 2003).

Estes resultados podem sugerir a hipótese de que as imagens sociais negativas associadas a estas famílias estejam relacionadas com 0 elevado número de crianças e adolescentes acolhidos e o tempo de institucionalização prolongado, tanto no Brasil como em Portugal, apesar da existência de vínculos familiares em grande parte dos casos (Constantino et al., 2013; ISS, 2014). As imagens sociais negativas associadas às famílias de crianças e adolescentes acolhidos podem levar ao desinvestimento dos profissionais em ações de restauração ou fortalecimento de vínculos para reinserção familiar, favorecendo a adoção do acolhimento institucional e o prolongamento desta medida (Constantino et al., 2013).

Os resultados apresentados também permitiram verificar que 0 contato com esta população tem alguma influência na imagem social acerca das famílias de crianças e jovens em acolhimento institucional, na medida em que os participantes com mais contato salientam mais atributos relativos às lacunas parentais em termos afetivos, funcionais e estruturais (e.g., negligente, desorganizada, desapegada emocionalmente) e menos aspetos relativos ao estado da família em si (e.g., triste, problemática) do que os participantes sem contato com esta população. Estudos futuros devem ser realizados com 0 intuito de explorar tais diferenças. No entanto, destaca-se que os participantes que possuem contato parecem considerar mais estas famílias com relação aos aspectos que afetam os filhos (aspectos funcionais e estruturais), e menos com relação às famílias em si mesmas (por exemplo, sentimentos e vínculos entre os membros), o que pode levar a uma desconsideração dos impactos do afastamento familiar para as próprias famílias, além do impacto no desenvolvimento dos filhos. Sabe-se que o afastamento familiar é uma medida extrema, que possui alto impacto emocional tanto para os filhos acolhidos como para as famílias em si, de forma que a intervenção deve considerar a situação em toda sua complexidade, aspecto que pode estar sendo negligenciado quando profissionais possuem imagens sociais altamente negativas sobre as famílias.

Cabe ressaltar algumas limitações deste estudo no que diz respeito aos aspectos metodológicos. Quanto aos participantes, sugere-se que novos estudos ampliem a amostra para outras regiões do país, já que no Brasil apenas o estado do Rio Grande do Sul participou da amostra e em Portugal, apenas a área geográfica da Grande Lisboa. Além disso, pesquisas com abordagem qualitativas podem ajudar a 
perceber aspectos históricos, sociais e culturais associados a tais imagens sociais e aprofundar o conhecimento sobre elas.

É ainda importante que novos estudos possam investigar o impacto destas imagens tanto nas crianças e jovens, como em suas famílias, no que diz respeito ao seu bem-estar, relacionamento com a equipe técnica do acolhimento e possibilidade de reinserção familiar, já que essas imagens podem influenciar comportamentos e atitudes frente a esta população. No Brasil e também em Portugal, há políticas públicas para reinserção de crianças e adolescentes na família de origem ou família extensa, mas as imagens sociais negativas, tanto de pessoas com contato quanto sem contato com essa população, podem dificultar os processos de reinserção familiar, por não haver crenças quanto às possibilidades e potencialidades de tais famílias receberem seus filhos de volta.

A partir deste estudo, foi possível dar visibilidade a um fenômeno pouco estudado empiricamente tanto no Brasil quanto em Portugal. Sugere-se, a partir destes resultados, intervenções que possibilitem a sensibilização não só de profissionais, mas também de pessoas que possuem alguma forma de contato com essas crianças e jovens e suas famílias, a fim de minimizar o impacto de tais imagens sociais negativas. Repensar e discutir, criticamente, aspectos históricos, sociais e culturais, também pode contribuir para uma conscientização a respeito das atitudes e práticas adotadas, assim como possibilitar 0 direcionamento do olhar para as potencialidades das crianças, adolescentes e suas famílias.

\section{Referências}

Brasil. (1990, 16 de julho). Lei no 8.069, de 13 de julho de 1990. Estatuto da Criança e do Adolescente. Diário Oficial da União, p. 13.563.

Brasil. (2009, 3 de agosto). Lei 12.010, de 3 de agosto de 2009. Diário Oficial da União, p. 1.

Brasil. Conselho Nacional dos Direitos da Criança e do Adolescente (CONANDA) e Conselho Nacional de Assistência Social (CNAS). (2006). Plano Nacional de Promoção, Proteção e Defesa do Direito de Crianças e Adolescentes à Convivência Familiar e Comunitária. Retrieved in February 23, 2014, from http://www.sdh.gov.br/assuntos/criancas-eadolescentes/programas/convivencia-familiar-e-comunitaria

Calheiros, M. M. (2006). A construção social do mau trato e negligência: do senso-comum ao conhecimento científico. Fundação Calouste Gulbenkian/Fundação para a Ciência e Tecnologia. Imprensa de Coimbra Lda. 
Calheiros, M. M., Garrido, M. V., Lopes, D., \& Patrício, J. N. (2015). Social images of residential care: How children, youth and residential care institutions are portrayed? Children and Youth Services Review, 55, 159-169. DOI: 10.1016/j.childyouth.2015.06.004

Calheiros, M. M., Graça, J., \& Patrício, J. N. (2014). From assessing needs to designing and evaluating programs: case study of a family support program in Portugal. Children and Youth Services Review, 36(1), 170-178.

Calheiros, M. M, Seabra, D., \& Fornelos, M. (1993). The nature and research of residential and foster care provision in Portugal. In Colton, M., \& Hellinckx, W., (Eds.). Child Care in Countries of the European Community (pp. 177-195). Avebury: Gower Publishing Group.

Carneiro, P., Albuquerque, P., \& Fernandez, A. (2008). Portuguese category norms for children. Behavior Research Methods, 40, 177-182.

Cavalcante, L. I. C., Costa Silva, S. S. da, \& Magalhães, C. M. C. (2010). Institucionalização e reinserção familiar de crianças e adolescentes. Revista Mal Estar e Subjetividade, 10(4), 11471172.

Constantino, P., Assis, S. G., \& Mesquita, V. S. F. (2013). Crianças, adolescentes e famílias em SAI. In S. G. Assis \& L. O. P. Farias (Eds). Levantamento nacional das crianças e adolescentes em serviço de acolhimento (pp. 161-220). São Paulo: Hucitec Editora.

Convenção Sobre os Direitos da Criança. Resolução da Assembleia da República [Convention on the Rights of the Child. Resolution of the Assembly of the Republic], no 20/90. D.R. no 211, $1^{\text {a Série, }}$ 12 de Setembro de 1990.

Corsini, R. J. (1999). The dictionary of psychology. Philadelphia, PA: Taylor \& Francis.

Goffman, E. (1988). Estigma: Uma análise sobre a situação da pessoa estigmatizada. Rio de Janeiro: Livros Técnicos e Científicos.

ISS (2014). CASA 2013 - Caracterização anual da situação de acolhimento das crianças e jovens. Retrieved in February 23, 2014, from http://www4.segsocial.pt/documents/10152/13326/Relatorio_CASA_2013

Kuznetsova, T. I. (2005). Social stereotypes of the perception of graduates of children's homes. Russian Education and Society, 47, 19-30.

Marzol, R. M., Bonafé, L., \& Yunes, M. A. M. (2012). As perspectivas de crianças e adolescentes em situação de acolhimento sobre os cuidadores protetores. Psico, 43(3), 317-324. 
Massey, S. G. (2010). Valued differences or benevolent stereotypes? Exploring the influence of positive beliefs on anti-gay and antilesbian attitudes. Psychology \& Sexuality, 1, 115-130. DOI: $10.1080 / 19419899.2010 .484593$

Nario-Redmond, M. R. (2010). Cultural stereotypes of disabled and non-disabled men and women: Consensus for global category representations and diagnostic domains. British Journal of Social Psychology, 49, 471-488. DOI: $10.1348 / 014466609 \times 468411$

Organização das Nações Unidas (1989). Convenção dos Direitos da Criança (adotada em 20 de novembro de 1989). Assembléia Nacional das Nações Unidas. Retrieved in January 14, 2014, from http://www.unicef.org/brazil/pt/resources_10120.htm

Portugal (1999). Diário da República - I Série A. Lei de protecção de crianças e jovens em perigo - Lei 147/99.

Portugal (2007). Diário da República, $2^{a}$ série, No 90, 10 de Maio de 2007, Despacho no 8393/2007.

Portugal (2012). Diário da República, 2a Série, No 128, 4 de Julho de 2012, Despacho n. ${ }^{\circ}$ 9016/2012.

Rizzini, I., \& Rizzini, I. (2004). A institucionalização de crianças no Brasil: Percurso histórico e desafios presentes. Rio de Janeiro: Ed. da PUC-RJ.

Rodrigo, M. J., Máiquez, M. L., Martín, J. C., \& Byrne, S. (2008). Preservación familiar. Un enfoque positivo para la intervención con familias. Madrid: Pirámide.

Rodrigues, S., Barbosa-Ducharne, M., \& del Valle, J. (2013). The quality of residential child care in Portugal and the example of its development in Spain. Papeles del Psicólogo, 34, 11-22.

Siqueira, A. C. (2012). A garantia ao direito à convivência familiar e comunitária em foco. Estudos de Psicologia, 29(3), 437-444. DOI: $10.1590 /$ S0103-166X2012000300013

Siqueira, A. C., \& Dell'Aglio, D. D. (2006). O impacto da institucionalização na infância e na adolescência: uma revisão de literatura. Psicologia \& Sociedade, 18(1), 71-80. DOI: 10.1590/S0102-71822006000100010

Siqueira, A. C., Massignan, L., \& Dell'Aglio, D. D. (2011). Reinserção familiar de adolescentes institucionalizados: Processos malsucedidos. Paidéia, 21(50), 383-391. DOI: 10.1590/S0103863X2011000300011

Siqueira, A. C., Tubino, C. L., Schwarz, C., \& Dell'Aglio, D. D. (2009). Percepção das figuras parentais na rede de apoio de crianças e adolescentes institucionalizados. Arquivos Brasileiros de Psicologia, 61(1), 176-190.

Sykes, J. (2011). Negotiating stigma: Understanding mothers responses to accusations of child neglect. Children and Youth 
Services Review, 33(3), 448-456. DOI: 10.1016/j.childyouth.2010.06.015

Vasconcelos, Q. A., Yunes, M. A. M., \& Garcia, N. M. (2009). Um estudo ecológico sobre as interações da família com o abrigo. Paidéia, 19(43), 221-229. DOI: 10.1590/S0103863X2009000200010

Yunes, M. A. M., \& Szymanski, H. (2003). Crenças, sentimentos e percepções acerca da noção de resiliência em profissionais da Saúde e Educação que atuam com famílias pobres. Psicologia da Educação, 17, 119-137.

\author{
Endereço para correspondência \\ Jana Gonçalves Zappe \\ Universidade Federal de Santa Maria - UFSM \\ Rua Evaristo Tonin, 382, Bairro Itararé, CEP 97045-180, Santa Maria - RS, Brasil \\ Endereço eletrônico: janazappe@hotmail.com
}

\title{
Naiana Dapieve Patias
}

Faculdade Meridional - IMED

Rua André Marques, 536 apto 202, Centro, CEP 97010-040, Santa Maria - RS, Brasil

Endereço eletrônico: naipatias@hotmail.com

\section{Joana Nunes Patrício}

Instituto Universitário de Lisboa - ISCTE-IUL

Avenida das Forças Armadas, Edifício ISCTE-IUL, CIS-IUL, Sala 2w17, CEP 1649026, Lisboa, Portugal

Endereço eletrônico: joana.nunespatricio@gmail.com

\section{Maria Manuela Calheiros}

Instituto Universitário de Lisboa - ISCTE-IUL

Avenida das Forças Armadas, Edifício ISCTE-IUL, CIS-IUL, Sala 2w17, CEP 1649026, Lisboa, Portugal

Endereço eletrônico: maria.calheiros@iscte.pt

\section{Margarida Vaz Garrido}

Instituto Universitário de Lisboa - ISCTE-IUL

Avenida das Forças Armadas, Edifício ISCTE-IUL, CIS-IUL, Sala 2w17, CEP 1649026, Lisboa, Portugal

Endereço eletrônico: margarida.garrido@iscte.pt

\section{Diniz Lopes}

Instituto Universitário de Lisboa - ISCTE-IUL

Avenida das Forças Armadas, Edifício ISCTE-IUL, CIS-IUL, Sala 2w17, CEP 1649026, Lisboa, Portugal

Endereço eletrônico: diniz.lopes@iscte.pt

\section{Débora Dalbosco Dell'Aglio}

Universidade Federal do Rio Grande do Sul - UFRGS

Rua Ramiro Barcelos, 2600, Bairro Santa Cecília, CEP 90035-003, Porto Alegre RS, Brasil

Endereço eletrônico: dddellaglio@gmail.com

Recebido em: 14/03/2016

Reformulado em: 02/11/2016

Aceito em: 02/11/2016 
Jana Gonçalves Zappe, Naiana Dapieve Patias, Joana Nunes Patrício, Maria Manuela Calheiros, Margarida Vaz Garrido, Diniz Lopes,

Débora Dalbosco Dell Aglio

\section{Notas}

* Professora Adjunta na Universidade Federal de Santa Maria (UFSM). Possui Graduação e Mestrado em Psicologia pela UFSM e Doutorado em Psicologia pela Universidade Federal do Rio Grande do Sul (UFRGS). Especialista em Psicologia Jurídica (CFP) e em Crianças e Adolescentes em Situação de Risco (UNIFRA). Participa do Núcleo de Estudos e Pesquisas em Adolescência (NEPA/UFRGS) e do Grupo de Trabalho Juventude, Resiliência e Vulnerabilidade da Associação Nacional de Pesquisa e Pós-Graduação na Psicologia (ANPEPP). Atuou como psicóloga na Fundação de Atendimento Socioeducativo do Rio Grande do Sul (FASE-RS) e na Secretaria de Saúde da Prefeitura Municipal de Santa Maria.

** Psicóloga, Mestre em Psicologia (UFSM), Doutora em Psicologia (UFRGS), Especialista em Criança e Adolescente em Situação de Risco (UNIFRA), Especialista em Psicologia Escolar (CAPE, Fato). Docente dos cursos de graduação e mestrado em Psicologia da Faculdade Meridional (IMED), Passo Fundo, RS, Brasil.

*** Licenciada em Psicologia e mestre em Psicologia Social e das Organizações. Iniciou a sua experiência profissional como assistente de investigação no Centro de Investigação e Intervenção Social (CIS-IUL), do ISCTE-IUL. Neste âmbito teve funções de conceção e avaliação de programas na área dos serviços de proteção a crianças e jovens em risco, especificamente ao nível dos maus tratos e negligência parental, dos serviços de acolhimento residencial, e da educação parental/familiar. Colaborou ainda em projetos na área da proteção de crianças na primeira infância, e na área da educação (pré-escolar, básico, secundário) como avaliadora e consultora externa. Atualmente, além da investigação na área da proteção a crianças e jovens em risco, trabalha como psicóloga numa creche e desenvolve projetos na área da prevenção de maus-tratos e da parentalidade positiva.

**** Doutorada em Psicologia Social Comunitária, ISCTE (2003), é Professora Auxiliar no Departamento de Psicologia Social e das Organizações do Instituto Universitário de Lisboa (ISCTE-IUL) onde tem leccionado várias Unidades Curriculares nos três ciclos de estudo. Os interesses de investigação na área da Psicologia aplicada enquadram-se teoricamente na psicologia social do desenvolvimento e intervenção comunitária, focam-se na construção do self e no bem-estar e nos factores de risco associados ao desenvolvimento de crianças e jovens em risco; mau trato e negligência parental; e desenho e avaliação de programas de intervenção com famílias e crianças em instituições, escolas e comunidade. Criou o Mestrado de Psicologia Comunitária e Protecção de Crianças e Jovens em Risco sendo a Directora do mesmo entre 2006 a 2013. Foi Presidente da Associação Portuguesa de Psicologia (2010 - 2013) e Directora do Departamento de Psicologia Social e das Organizações (2012 - 2014). Apresenta fortes relações com a comunidade tendo trabalhado em projectos de investigação e programas de intervenção baseados em teoria e investigação para o Governo (a nível nacional e do Conselho da Europa), e ONGs. Com 80 publicações científicas, 44 das quais em revistas e livros internacionais indexados, a vertente aplicada da sua investigação aos problemas psicossociais tem motivado a publicação nacional de vários livros e artigos para a comunidade académica, técnica e de ensino.

***** Doutorada em Psicologia Social e professora no Departamento de Psicologia social e das Organizações do ISCTE-IUL onde coordena e leciona unidades curriculares de métodos e de psicologia em cursos de licenciatura, mestrado e doutoramento e orienta estágios e teses de mestrado e doutoramento. É investigadora no CIS-IUL onde desenvolve pesquisa na área da cognição social. $\mathrm{Na}$ sua investigação atual explora os processos corporalizados subjacentes à utilização de uma primeira ou segunda língua. Outros interesses de investigação incluem Cognição Socialmente Distribuída nomeadamente a influência do contexto físico e social na cognição: 1) perceção de pessoas; 2) ancoragem de conceitos abstratos (valência, tempo, política); 3) codificação e recordação colaborativa. O seu trabalho 
de investigação tem resultado em diversas publicações científicas. Tem ainda colaborado em projetos de intervenção organizacional e social.

****** Professor Auxiliar no Departamento de Psicologia Social e das Organizações do ISCTE-IUL e Investigador Integrado do CIS-IUL. Completou o seu doutoramento em Psicologia Social em 2007 e foi bolseiro Pos-Doc da FCT nas Universidades Paris Ouest Nanterre la Défense, Paris Descartes e Universidade do Porto entre 2010 e 2013. É presentemente Editor Associado do International Journal of Conflict and Violence, e presidente da Associação Portuguesa de Psicologia (APP). A sua investigação tem-se desenvolvido presentemente em torno do estudo das relações interpessoais, especialmente das relações amorosas homo e heterossexuais, sua manutenção ou dissolução e derrogação de alternativas à relação; dos processos de validação do conhecimento produzido em grupo, nomeadamente o processamento de informação proveniente de decisões grupais e atribuição de credibilidade ou validade a esta informação; das metodologias quantitativas e sua aplicação em Psicologia, com particular enfoque no desenvolvimento e estudo de medidas psicológicas e determinação das suas qualidades psicométricas. Paralelamente às suas atividades científicas, exerce também atividades pedagógicas, i.e., de ensino universitário, lecionando cadeiras de $10,2^{\circ}$ e $3^{\circ}$ ciclos, com destaque para a Psicometria e os Métodos de Investigação Quantitativos.

$* * * * * * *$ Psicóloga, mestre e doutora em Psicologia. Docente do Programa de PósGraduação em Psicologia da Universidade Federal do Rio Grande do Sul. 